\title{
Physical exercise and the patient with schizophrenia
}

CPD

Margaretha Viljoen, Johannes L Roos

\section{Background \\ Schizophrenia is a severe mental disorder with profound effects on a person's life. In addition to the psychiatric symptoms, patients with schizophrenia generally have multiple somatic comorbidities, such as cardiovascular and metabolic disorders. The general practitioner (GP) is of key importance for the patient's continuous care and holistic wellbeing.}

\section{Objective}

The aim of this article is to emphasise the role of GPs in embracing physical exercise as add-on treatment to antipsychotic medications, and to illustrate the value of exercise for people with schizophrenia by summarising the effects on the psychiatric symptoms, neuroanatomical and neurochemical characteristics, and general physiological and psychological health.

\section{Discussion}

Physical exercise can lead to improvements in positive, negative and cognitive symptoms, as well as in somatic comorbidities, global functioning and quality of life. Physical exercise can be a valuable add-on intervention for people with schizophrenia. The GP is essential for prescribing and following up on exercise tailored for the individual.
SCHIZOPHRENIA is a severe mental disorder with profound effects on a person's health and quality of life. Psychiatric symptoms present predominantly as positive symptoms (delusions and hallucinations), negative symptoms (lack of motivation, low energy levels, reduction in spontaneous speech, social withdrawal and affective flattening) and impaired neurocognitive functioning (perception, memory, attention and executive function). Antipsychotic medications, as the first line of therapy, usually decrease the positive symptoms, while their effect on negative and cognitive symptoms is limited. Unfortunately, approximately $30 \%$ of patients with schizophrenia are refractory to antipsychotic medication. ${ }^{1}$

Largely because of multiple somatic comorbidities, schizophrenia is increasingly recognised as a systemic disorder rather than just a mental disorder. In addition to the psychiatric symptoms, individuals with schizophrenia are at risk of a number of physical diseases such as cardiovascular and respiratory illnesses, metabolic disorders such as type 2 diabetes, and several immunerelated disturbances, contributing to a life expectancy 15-25 years lower than that in the general population. ${ }^{1}$ Several factors are implicated in this burden of physical comorbidity, including

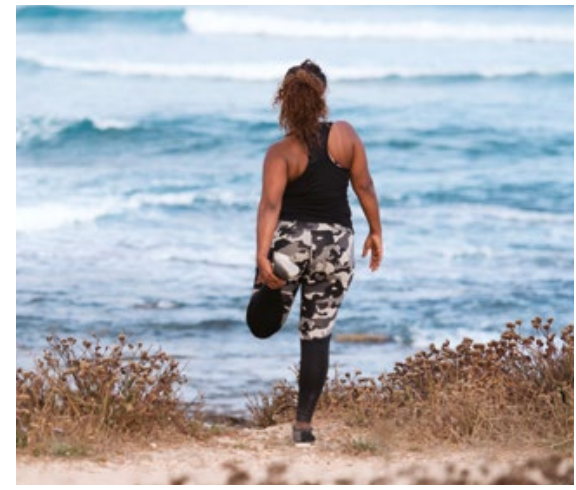

antipsychotic medication, adverse prenatal neurodevelopment and modifiable lifestyle risks such as poor diet, smoking and lack of physical activity. ${ }^{2,3}$ More recently, evidence is surfacing for a shared genetic risk, including overlapping genes, between psychotic disorders and some of the somatic disorders seen in schizophrenia. ${ }^{2,3}$ This may explain the fact that young patients with schizophrenia could have a variety of physical somatic disorders before the onset of the first psychotic episode. $^{3}$

Alterations in many neurobiological and humoral factors have been studied as causal factors underlying the pathophysiology of schizophrenia, including volume and functional deficits of cerebral white and grey matter, attenuated resting state connectivity and task-related activation of certain brain areas; $;^{4,5}$ several neurotransmitter systems; growth factors, including brain-derived neurotrophic factor (BDNF); and immunerelated mechanisms, especially those related to pro-inflammatory activity. ${ }^{5,6}$ Inconsistencies in results confound absolute conclusions, but volumetric and functional deficits of the hippocampus (a brain structure central to learning, memory and cognitive integration) and a decrease in the levels of BDNF (a protein involved in the neuroplasticity 
of the central nervous system) are repeatedly reported. In addition to the neurobiological and humoral factors, polygenetic risk is described as a possible causal factor contributing to both the psychiatric symptoms and comorbidities. ${ }^{6-8}$

Most individuals with schizophrenia have reduced rates of physical activity compared with the general population. Major contributors to this sedentary behaviour include fatigue, partly linked to the effects of antipsychotic medication; somatic comorbidities such as obesity, metabolic and cardiorespiratory health issues; low motivation to exercise, probably related to the negative symptoms of the disorder; lack of confidence, especially anxiety and stress about exercising in public; and a lack of resources or encouragement from others to exercise. ${ }^{9,10}$

In the general population, sedentary behaviour was shown to be independently related to increased risks for the development of cardiovascular disease, type 2 diabetes and all-cause mortality. ${ }^{9}$ Associations also exist between sedentary behaviour and the risk for somatic disorders in patients with schizophrenia. ${ }^{9,11,12}$ Negative associations likewise exist between sedentary lifestyles and mental health, both in the general population and in people with mental health issues. ${ }^{7,9}$ In fact, physical inactivity and poor fitness have particularly strong relationships with negative symptoms and cognitive deficits in schizophrenia. ${ }^{13}$ In addition, it is now known that physical inactivity is epigenetically harmful. ${ }^{7}$ While the genome of an organism is relatively stable, its expression (phenotype) is influenced by many epigenetic factors. ${ }^{5}$ Evidence exists, not only for the benefit of physical exercise as an epigenetic modulator of brain plasticity and cognition, ${ }^{7,14}$ but also for somatic disturbances such as exercise-induced epigenetic modifications in genes related to insulin resistance and type 2 diabetes, inflammation, cardiovascular disease and obesity. ${ }^{15}$

The aim of this article is to provide general practitioners (GPs) and primary healthcare workers with an overview of the benefits of physical exercise as an add-on therapy to pharmacological intervention for patients with schizophrenia.

A literature search was performed of databases and by hand. Databases included EBSCOhost Research Interface databases; American Psychological Association (APA) PsycInfo; Academic Search Complete; Africa-Wide Information; Cumulative Index to Nursing and Allied Health Literature (CINAHL); E-Journals; MEDLINE and APA

PsycArticles. Because of the large number of articles, Table 1 was compiled from systematic reviews and meta-analyses from 2015 to the present, allowing for the inclusion of results obtained from thousands of patients. The results relate to the effects of physical exercise on psychiatric symptoms, neuroanatomical and neurochemical characteristics, and general physiological and psychological health of patients with schizophrenia.

\section{Discussion}

Numerous studies confirmed that exercise, as an add-on to pharmacological therapy, can significantly attenuate the psychiatric symptoms of schizophrenia and improve the quality of life, general cognition and global functioning of patients with this disorder (Table 1). In addition, physical exercise is seen to be a valuable tool in the treatment of the multiple cardiorespiratory, metabolic and other comorbidities associated with schizophrenia. Beneficial effects have also been found on the neural correlates of psychiatric symptoms, but the exercise-induced effects were almost always smaller than in healthy controls, and minor inconsistencies exist between results from different studies (Table 1). It is feasible to assume that differences in exercise regimens, cohorts and individual risk factors played a part in the variations. For instance, there are indications that disrupted neuroplasticity in schizophrenia may underlie structural and functional alterations as well as the response to exercise. ${ }^{8,16}$ This is thought to be related to patients' burden of polygenic risk, which modulates biological processes involved with synaptic ion channel activity, calcium and glutamate signalling, and regulation of cell morphogenesis. ${ }^{8,17}$ Physical exercise is suggested to improve neuroplasticity through the regulation of central growth factors, and evidence points to BDNF as the potential underlying mechanism. ${ }^{18}$

\section{Types and intensity of exercise}

The quest to promote physical activity among people with serious mental illness is nothing new. Varying degrees of success have been reported, with both structured, facility-based programs and moderate lifestyle activity changes such as the introduction of regular walking. 9,19 However, the minimum dosage of exercise and method of intervention for patients with schizophrenia remains a fundamental issue. Guidelines from the European Psychiatric Association (EPA) on physical activity for severe mental illness suggest aerobic exercise of moderate-to-vigorous intensity at a frequency of 2-3 times a week, achieving 90-150 minutes of moderate-to-vigorous physical activity per week. ${ }^{9}$ Dropout rates of approximately $27 \%$ have been reported, but indications are that the dropout rate can be lowered by motivation and supervision by exercise professionals. ${ }^{9}$ Despite the prominence of the aforementioned guidelines, this level of activity may be excessive, and in some cases even risky, for those patients with schizophrenia who previously had overtly sedentary lifestyles and/or those whose physical health is seriously compromised by somatic comorbidities. It is also unlikely that one prescription would be suitable for all patients. It seems more logical that exercise prescriptions for patients with schizophrenia should, like other medical prescriptions, be tailored to each patient's needs and revised on a regular basis. A series of articles in a recent edition of Australian Journal of General Practice addressed exactly this issue, as it applies to physical morbidities. In short, the edition covers important concepts in prescribing exercise tailored to the individual's needs and baseline health, and argues that exercise prescriptions should be treated in a similar way as the prescription of medication. Rather than reiterating the information, the authors recommend that those who are interested read at least three of the articles. ${ }^{20-22}$ 
Table 1. Effects of physical exercise on the psychiatric symptoms, neuroanatomical and neurochemical characteristics, and general physiological and psychological health of patients with schizophrenia

\section{Studies \\ Systematic review and meta-analysis of 10 trials (385 patients). Analysis of cognitive outcomes. ${ }^{29}$}

Systematic review of seven studies (389 patients). Analysis of effects on symptoms, strength and CVS fitness. ${ }^{12}$

Systematic review and metaanalysis of longitudinal studies $(30,025$ patients). Analysis of psychosis prevention. ${ }^{35}$

Systematic review of six studies (300 patients) Analysis of PANSS. ${ }^{25}$

Systematic review and metaanalysis of 29 studies (1109 patients). Analysis of effects on schizophrenia symptoms. ${ }^{30}$

\section{Exercise characteristics}

Aerobic exercise: 12.2 (4-24) weeks; 2.9 (2-4) times/week; 20-60 min/ session.

Combined exercise for $\geq 12$ weeks, average of 95 minutes of aerobic plus strength training, two days/week.

Self-reported high versus low levels of activity. Baselines at between nine and 18 years. Follow-up between four and 32 years after baseline.

Mild-to-moderate physical activity $\geq 150 \mathrm{~min} /$ week, such as yoga, martial arts, sports, resistance training.

Aerobic exercise, anaerobic exercise and yoga. Approximately 360-720 min/week. Total duration approximately 12 weeks.

\section{Effects of exercise intervention}

Significantly improved working memory, social cognition and attention/vigilance. Effects on processing speed, verbal and visual memory and problem solving not significant. Greater effects with higher exercise dosage and exercise professional involvement.

Significant improvements in schizophrenia symptoms, overall mental health and muscle strength (leg, bicep curl, chest press, leg press). Improvements in CVS fitness were clinically, but not statistically, meaningful.

Initial analyses suggested that physical activity may be a protective factor against the emergence of psychosis/ schizophrenia, but when adjusting for covariates, the association was no longer significant.

Physical activity significantly improved aggregate total PANSS score and positive symptoms PANSS score. Negative symptoms PANSS score showed non-significant trend toward improvement.

Exercise was superior to control conditions in improving total symptom severity, negative and general symptoms, quality of life, global functioning and depressive symptoms. Recommendation: supervised group exercise, three times/week, $30 \mathrm{~min} / \mathrm{session}$, for a minimum of 12 weeks.
Meta-review of seven systematic reviews and meta-analyses. Analysis of position statement of European Psychiatric Association. ${ }^{9}$
Moderate-to-vigorous physical activity. Conclusions mainly based on aerobic exercise.
Reduced total, negative and positive symptoms. Body mass index results inconsistent. Cardiorespiratory fitness increases over 12 weeks. Increase in global cognition, working memory and attention. Recommended: 90-150 minutes of moderate-tovigorous aerobic exercise/week. Better results with higher activity and supervision by exercise professionals.

Nine of 12 studies showed increases in BDNF. All studies of $\geq 8$ weeks showed positive cognitive effects (increases in global cognitive performance, working memory, attention-vigilance, response inhibition, etc).
Review of 12 studies on BDNF. ${ }^{6}$ Aerobic exercise between 30 min acute and 12 weeks $3 \times 60 \mathrm{~min} /$ week.

Systematic review of nine studies (168 patients). Analysis of exercise-induced neural effects. ${ }^{17}$

Review of exercise-induced changes in brain structure $(n=7)$ and biomarkers $(n=7)$. Analysis of neurotrophins, oxidative and inflammatory. ${ }^{36}$
Heterogeneous type, frequency, duration and intensity of exercise.

Aerobic and high-intensity interval training including moderate aerobic and combined aerobic-resistance.
Increase in hippocampal volume, dependent on exercise. Increases seen in volume of temporal gyri, extrastriate body area activation, total cerebral matter volume and white matter integrity in fibre tracts, need replication.

Increases in hippocampal volume correlated with an increase in short-term memory, linked to cardiorespiratory fitness and exercise capacity. An increase in total cerebral volume and improvement in fibre tracts correlated with increased cardiorespiratory fitness. Increased BDNF, correlated with cognitive improvements. Cognitive benefits may be related to BDNF-induced neurogenesis.
Review of neurobiological effects of aerobic exercise on patients with schizophrenia. ${ }^{5}$
Focus on relatively long-term aerobic training.
Hippocampal and cortical volume increases seen after endurance training. Three months of endurance training increased motor cortical inhibition. Six months of endurance training increased integrity in white matter fibre tracts related to motor function. Cognitive improvements correlated with increases in BDNF levels.

Functional brain changes, especially in the frontal lobe, accompanied by improvements in executive functions. Lower white matter atrophy and smaller white matter lesions.
Review of 18 studies. Analysi of exercise-induced brain changes. $^{37}$
Resistance exercises performed 1-3 days/week; 30-90 min/ session; 9-52 weeks. 
Table 1. Effects of physical exercise on the psychiatric symptoms, neuroanatomical and neurochemical characteristics, and general physiological and psychological health of patients with schizophrenia (cont'd)

\begin{tabular}{|c|c|c|}
\hline Studies & Exercise characteristics & Effects of exercise intervention \\
\hline $\begin{array}{l}\text { Systematic review and meta- } \\
\text { analysis of } 20 \text { studies. Analysis } \\
\text { of effects on comorbidities. }{ }^{38}\end{array}$ & $\begin{array}{l}\text { On average, } 90 \text { minutes of moderate- } \\
\text { to-vigorous exercise per week. }\end{array}$ & $\begin{array}{l}\text { This amount of exercise was reported to significantly improve } \\
\text { functioning, somatic comorbid disorders and neurocognition. }\end{array}$ \\
\hline $\begin{array}{l}\text { Review of effects on metabolic } \\
\text { syndrome and cardiorespiratory } \\
\text { fitness. }^{39}\end{array}$ & $\begin{array}{l}\text { Continuous endurance (13 studies) } \\
\text { and high-intensity interval training } \\
\text { (five studies). }\end{array}$ & $\begin{array}{l}\text { High-intensity interval endurance training may be more beneficial } \\
\text { than continuous endurance training. }\end{array}$ \\
\hline $\begin{array}{l}\text { Systematic review and } \\
\text { meta-analysis of } 17 \text { trials ( } 954 \\
\text { patients). Analysis of effects on } \\
\text { negative symptoms. }{ }^{40}\end{array}$ & $\begin{array}{l}\text { Twelve aerobic RCTs and five } \\
\text { non-aerobic RCTs. }\end{array}$ & $\begin{array}{l}\text { Aerobic, but not non-aerobic interventions, reduced the negative } \\
\text { and positive symptoms. Sensitivity analysis on studies with a low } \\
\text { risk of bias confirmed the effect on negative, but not on positive, } \\
\text { symptoms. }\end{array}$ \\
\hline
\end{tabular}

BDNF, brain-derived neurotrophic factor; CVS, cardiovascular system; PANSS, Positive and Negative Syndrome Scale; RCT, randomised controlled trial

Individuals with schizophrenia have lower levels of aerobic capacity than the general population..$^{23}$ There is evidence that both their mental and physical health improve with increases in their aerobic capacity. ${ }^{23}$ For instance, evidence exists for correlations between maximum aerobic capacity $\left(\mathrm{VO}_{2} \max \right)$ and global functioning, ${ }^{24}$ between improvements in aerobic capacity and improvements in the general psychopathology subscale and total score on the Positive and Negative Syndrome Scale (PANSS), ${ }^{23}$ and between increases in aerobic fitness (ie increased $\mathrm{VO}_{2}$ max and $\mathrm{VO}_{2}$ peak) and clinical improvements and reduction in mortality. ${ }^{24}$ This suggests that any physical activity that improves aerobic capacity/ aerobic fitness - including sports, martial arts, yoga and dance - may potentially also improve the psychiatric symptoms and comorbidities of schizophrenia. In fact, several studies have shown such benefits..$^{25-27}$ Better results are generally reported with higher intensity exercise, but dropout rates and the potential for adverse events in high-risk individuals may increase. ${ }^{9,19}$

Various studies support early intervention in schizophrenia, even before the first psychotic episode. For example, young patients can have several physical disorders before the onset of the first psychotic episode, ${ }^{3}$ and multimorbidity increases over the psychosis spectrum, with a stronger association between multimorbidity and psychosis among younger adults. ${ }^{28}$

\section{Motivation to exercise}

A problem central to prescribing exercise for patients with schizophrenia is that most have reduced levels of physical activity. Contributors to this sedentary behaviour and the potential implications for somatic and mental health were discussed in the introduction. Several studies address possible ways to overcome this reluctance or these barriers to exercise. Significantly better results are generally reported with supervision by exercise professionals. ${ }^{29,30}$ Social support from health professionals such as doctors, nurses and psychologists, as well as from family members, friends and peers, plays a significant part in the initiation of exercise and in compliance and adherence to exercise regimens. ${ }^{27}$ It is thus self-evident that tailored exercise regimens, accompanied by ongoing assessment, encouragement and adjustment, as described for somatic disorders, ${ }^{20-22}$ may motivate patients with schizophrenia to become physically active. In addition to conventional physical exercise, a number of studies showed that video games that require physical movement ${ }^{31}$ and physical exercise paired to tablet-based neurofeedback can motivate patients and consequently decrease the attrition rate. ${ }^{32}$ Relatively simple forms of wearable technology such as step counters may encourage a more active lifestyle and facilitate exercise prescriptions, ${ }^{19,21}$ while more sophisticated wearable technology may be employed to track patient progress.

\section{The general practitioner and exercise in schizophrenia}

Most patients with serious psychiatric disorders such as schizophrenia ideally would have at least intermittent psychiatrist support from mental health services. However, this is precluded in most low- and middle-income countries because of a shortage of access to such services. South Africa, for instance, has no more than 700 specialist psychiatrists for a population of 55 million. The majority practise in private, urban and peri-urban areas, while in the public rural primary healthcare sector, psychiatrists are employed at a rate of 0.03 per 100,000 population. ${ }^{33}$ Regardless of the availability of mental health services, the GP is essential for the ongoing care 
and holistic health of the patient with schizophrenia. An Australian study showed that $>88 \%$ of people with a psychotic illness consult a GP at least annually, and that for at least one-third of people living with schizophrenia in Australia, ongoing management is provided by their GP alone. ${ }^{34}$ The burden of physical comorbidities in schizophrenia and the inclusion of exercise as an add-on treatment to antipsychotic medications render follow-up of such patients by GPs of particular importance - especially in patients with cardiorespiratory comorbidities for whom inappropriate initiation of strenuous exercise could be of concern.

\section{Conclusion}

Beneficial effects of physical activity have been reported with exercise protocols that vary widely in terms of type of exercise, intensity and time per week spent exercising. On the basis of a meta-review of seven systematic reviews and meta-analyses, the European Psychiatric Association suggests between 90 and 150 minutes of moderate-to-vigorous aerobic exercise per week for people with schizophrenia spectrum disorders. ${ }^{9}$ Better results and lower dropout rates are reported with the inclusion of motivational influences and supervision by exercise professionals..$^{9,29,30}$ Although the value of the information derived from cohort studies and systematic reviews and meta-analyses cannot be overestimated, it is feasible to assume that the majority of patients with schizophrenia would best benefit from individually tailored exercise interventions. The GP, an important figure in the continuous care and holistic wellbeing of the patient with schizophrenia, has a number of roles to play. The GP can:

- develop appropriate individualised protocols, taking into consideration the patient's age, gender, general health status, physical fitness level, barriers to exercise (including somatic illness-related, socioeconomic and psychological barriers), as well as the personal activity preferences of the individual
- provide ongoing motivation and encouragement and, where necessary, involve the services of exercise professionals

- regularly monitor patients and provide feedback and, where appropriate, revise the physical activity regimen.

\section{Authors}

Margaretha Viljoen PhD, Professor in Psychiatry, Department of Psychiatry, Faculty of Health Sciences, The University of Pretoria, Pretoria, South Africa. mviljoen@webafrica.org.za

Johannes L Roos MMed (Psych), MD, Professor in Psychiatry, Department of Psychiatry, Faculty of Health Sciences, The University of Pretoria, Pretoria, South Africa

\section{Acknowledgements}

The authors would like to thank Mrs Erna Fourie of the Department of Psychiatry and Mrs Leonora Wydeman of the Medical Library, The University of Pretoria, Pretoria, South Africa.

Competing interests: None.

Funding: None.

Provenance and peer review: Not commissioned, externally peer reviewed.

\section{References}

1. Sadock BJ, Sadock VA, Ruiz P. Schizophrenia spectrum and other psychotic disorders. In: Kaplan and Sadock's synopsis of psychiatry behavioral sciences/clinical psychiatry. 11th edn. Philadelphia, PA: Wolters \& Kluwer, 2015.

2. Dieset I, Andreassen OA, Haukvik UK. Somatic comorbidity in schizophrenia: Some possible biological mechanisms across the life span. Schizophr Bull 2016;42(6):1316-19. doi: 10.1093/ schbul/sbw028.

3. Šimunović Filipčić I, Filipčić I. Schizophrenia and physical comorbidity. Psychiatr Danub 2018;30(Suppl 4):152-57.

4. Vanes LD, Mouchlianitis E, Patel K, et al. Neural correlates of positive and negative symptoms through the illness course: An fMRI study in early psychosis and chronic schizophrenia. Sci Rep 2019;9(1):14444. doi: 10.1038/s41598-019-51023-0.

5. Maurus I, Hasan A, Röh A, et al. Neurobiological effects of aerobic exercise, with a focus on patients with schizophrenia. Eur Arch Psychiatry Clin Neurosci 2019;269(5):499-515. doi: 10.1007/ s00406-019-01025-w.

6. Gökçe E, Güneş E, Nalçaci E. Effect of exercise on major depressive disorder and schizophrenia: A BDNF focused approach. Noro Psikiyatr Ars 2019;56(4):302-10. doi: 10.29399/npa.23369.

7. Di Liegro CM, Schiera G, Proia P, Di Liegro I. Physical activity and brain health. Genes (Basel) 2019;10(9):720. doi: 10.3390/genes10090720.

8. Papiol S, Popovic D, Keeser D, et al. Polygenic risk has an impact on the structural plasticity of hippocampal subfields during aerobic exercise combined with cognitive remediation in multi-episode schizophrenia. Transl Psychiatry 2017;7(6):e1159. doi: 10.1038/tp.2017.131.

9. Stubbs B, Vancampfort D, Hallgren M, et al. EPA guidance on physical activity as a treatment for severe mental illness: A meta-review of the evidence and position statement from the European psychiatric association (EPA), supported by the international organization of physical therapists in mental health (IOPTMH) Eur Psychiatry 2018;54:124-44. doi: 10.1016/j. eurpsy.2018.07.004

10. Yung AR, Firth J. How should physical exercise be used in schizophrenia treatment? Expert Rev Neurother 2017;17(3):213-14 doi: 10.1080/14737175.2017.1275571.

11. Bueno-Antequera J, Oviedo-Caro MÁ Munguia-Izquierdo D. Relationship between objectively measured sedentary behavior and health outcomes in schizophrenia patients: The PsychiActive project. Schizophr Res 2018;197:87-92. doi: 10.1016/j. schres.2017.11.022

12. Martin H, Beard S, Clissold N, Andraos K, Currey L. Combined aerobic and resistance exercise interventions for individuals with schizophrenia: A systematic review. Ment Health Phys Act 2017;12:147-55. doi: 10.1016/j. mhpa.2017.04.003.

13. Kimhy D, Lauriola V, Bartels MN, et al. Aerobic exercise for cognitive deficits in schizophrenia The impact of frequency, duration, and fidelity with target training intensity. Schizophr Res 2016;172(1-3):213-15. doi: 10.1016/j. schres.2016.01.055

14. Fernandes J, Arida RM, Gomez-Pinilla F. Physical exercise as an epigenetic modulator of brain plasticity and cognition. Neurosci Biobehav Rev 2017;80:443-56. doi: 10.1016/j. neubiorev.2017.06.012.

15. Barrón-Cabrera E, Ramos-Lopez O González-Becerra K, et al. Epigenetic modifications as outcomes of exercise interventions related to specific metabolic alterations: A systematic review. Lifestyle Genom 2019;12(1-6):25-44. doi: 10.1159/000503289.

16. Bhandari A, Voineskos D, Daskalakis ZJ, Rajji TK, Blumberger DM. A review of impaired neuroplasticity in schizophrenia investigated with non-invasive brain stimulation. Front Psychiatry 2016;7:45. doi: 10.3389/fpsyt.2016.00045.

17. Van der Stouwe ECD, van Busschbach JT, de Vries B, Cahn W, Aleman A, Pijnenborg GHM. Neural correlates of exercise training in individuals with schizophrenia and in healthy individuals: A systematic review. Neuroimage Clin 2018;19:287-301. doi: 10.1016/j.nicl.2018.04.018.

18. Jahshan C, Rassovsky Y, Green MF. Enhancing neuroplasticity to augment cognitive remediation in schizophrenia. Front Psychiatry 2017;8:191. doi: 10.3389/fpsyt.2017.00191.

19. Richardson CR, Faulkner G, McDevitt J, Skrinar GS, Hutchinson DS, Piette JD. Integrating physical mental health services for persons with serious mental illness. Psychiatr Serv 2005;56(3):324-31. doi: 10.1176/appi.ps.56.3.324

20. Margolis SA. Editorial: Exercise is medicine Moving an idea into action. Aust J Gen Pract 2020;49(4):165. doi: 10.31128/AJGP-04-20-1234e.

21. Orchard JW. Prescribing and dosing exercise in primary care. Aust J Gen Pract 2020;49(4):182-86. doi: 10.31128/AJGP-10-19-5110.

22. Williams A, Radford J, O'Brien J, Davison K. Type 2 diabetes and the medicine of exercise. The role of general practice in ensuring exercise is part of every patient's plan. Aust J Gen Pract 2020;49(4):189-93. doi: 10.31128/AJGP-09-19-5091.

23. Curcic D, Stojmenovic T, Djukic-Dejanovic S, et al. Positive impact of prescribed physical activity on symptoms of schizophrenia: Randomized clinical trial. Psychiatr Danub 2017;29(4):459-65. doi: 10.24869/psyd.2017.459. 
24. Vancampfort D, Guelinckx H, Probst M, et al. Aerobic capacity is associated with global functioning in people with schizophrenia. J Ment Health 2015;24(4):214-18. doi: 10.3109/09638237.2015.1036974.

25. Tarpada SP, Morris MT. Physical activity diminishes symptomatic decline in chronic schizophrenia: A systematic review. Psychopharmacol Bull 2017;47(4):29-40.

26. Soundy A, Roskell C, Stubbs B, Probst M, Vancampfort D. Investigating the benefits of sport participation for individuals with schizophrenia: A systematic review. Psychiatr Danub 2015;27(1):2-13.

27. Gross J, Vancampfort D, Stubbs B, Gorczynski P, Soundy P. A narrative synthesis investigating the use and value of social support to promote physical activity among individuals with schizophrenia. Disabil Rehabil 2016;38(2):123-50. doi: 10.3109/09638288.2015.1024343.

28. Stubbs B, Koyanagi A, Veronese N, et al. Physical multimorbidity and psychosis: Comprehensive cross-sectional analysis including 242,952 people across 48 low- and middle-income countries. BMC Med 2016;14(1):189. doi: 10.1186/s12916-0160734-z.

29. Firth J, Stubbs B, Rosenbaum S, et al. Aerobic exercise improves cognitive functioning in people with schizophrenia: A systematic review and meta-analysis. Schizophr Bull 2017;43(3):546-56 doi: 10.1093/schbul/sbw115.

30. Dauwan M, Begemann MJH, Heringa SM, Sommer IE. Exercise improves clinical symptoms, quality of life, global functioning, and depression in schizophrenia: A systematic review and metaanalysis. Schizophr Bull 2016;42(3)588-99. doi: $10.1093 /$ schbul/sbv164

31. Leutwyler H, Hubbard EM, Dowling GA. Adherence to a videogame-based physical activity program for older adults with schizophrenia. Games Health J 2014;3(4):227-33. doi: 10.1089/ g4h.2014.0006

32. Choi J, Taylor B, Fiszdon JM, et al. The synergistic benefits of physical and cognitive exercise in schizophrenia: Promoting motivation to enhance community effectiveness. Schizophr Res Cogn 2020;19:100147. doi: 10.1016/j.scog.2019.100147.
33. De Kock JH, Pillay BJ. A situation analysis of psychiatrists in South Africa's rural primary healthcare settings. Afr J Prim Health Care Fam Med 2017;9(1):1335. doi: 10.4102/phcfm.v9i1.1335.

34. Harrison C, Charles J, Britt H. Comorbidities and risk factors among patients with schizophrenia. Aust Fam Physician 2015;44(11):781-83.

35. Brokmeier LL, Firth J, Vancampfort D, et al. Does physical activity reduce the risk of psychosis? A systematic review and metaanalysis of prospective studies. Psychiatry Res 2020;284:112675. doi: 10.1016/j. psychres.2019.112675.

36. Firth J, Cotter J, Carney R, Yung AR. The pro-cognitive mechanisms of physical exercise in people with schizophrenia. Br J Pharmacol 2017:74(19):3161-72. doi: 10.1111/bph.13772.

37. Herold F, Törpel A, Schega L, Müller NG. Functional and/or structural brain changes in response to resistance exercises and resistance training lead to cognitive improvements A systematic review. Eur Rev Aging Phys Act 2019;16:10. doi: 10.1186/s11556-019-0217-2.

38. Firth J, Cotter J, Elliott R, French P, Yung AR. A systematic review and meta-analysis of exercise interventions in schizophrenia patients. Psychol Med 2015;45(7):1343-61. doi: 10.1017/ S0033291714003110.

39. Schmitt A, Maurus I, Rossner MJ, et al. Effects of aerobic exercise on metabolic syndrome, cardiorespiratory fitness, and symptoms in schizophrenia include decreased mortality. Front Psychiatry 2018;21;9:690. doi: 10.3389/ fpsyt.2018.00690.

40. Sabe M, Kaiser S, Sentissi O. Physical exercise for negative symptoms of schizophrenia: Systematic review of randomized controlled trials and metaanalysis. Gen Hosp Psychiatry 2020;62:13-20. doi: 10.1016/j.genhosppsych.2019.11.002. 